\title{
The Relationship between Reoperative Prognostic Nutritional Index Levels and Risk of In-Stent Restenosis following Cerebrovascular Bare-Metal Stent Implantation
}

\author{
Qiang Fang ${ }^{1}$, Yun Yang ${ }^{2}$, Jiajun Wei ${ }^{1}$, Ligen Fan ${ }^{1}$, Zhuoxi Xie ${ }^{1}$, Zhouming Zhu ${ }^{1}$ and Hao Ii $^{{ }^{*}}$ \\ ${ }^{1}$ Department of Neurology, The Affiliated Hospital of Guilin Medical University, Guilin, China \\ ${ }^{2}$ Department of Neurology, The Second Affiliated Hospital of Guilin Medical University, Guilin, China
}

*Corresponding author: Hao Li, Department of Neurology, The Affiliated Hospital of Guilin Medical University, Lequn Road, Guilin, Guangxi, 541000, China, Tel: 185-078-38866

\begin{abstract}
Introduction: In-stent restenosis (ISR) is a key parameter influencing long-term stent use in patients that have undergone cerebrovascular stent implantation, and it additionally impacts surgical treatment outcomes. Inflammation is a key driver of ISR incidence, To date, however, no studies have evaluated the relationship between ISR and prognostic nutritional index (PNI), which is based upon lymphocyte counts and serum albumin levels and is closely linked with inflammatory activity. In this study, we therefore assessed whether PNI levels upon admission were predictive of ISR incidence.
\end{abstract}

Methods: In total, 141 patients with a history of intracranial artery stent implantation (19.9\% female) were analyzed in this study. ISR was diagnosed based upon a $>50 \%$ narrowing of the blood vessel at the site of stenting relative to the diameter following the most recent intervention. Based on these criteria, patients were separated into ISR and restenosis-free groups.

Results: ISR was observed in 36 patients (25.5\%). The PNI of ISR patients upon admission was significantly lower than that of restenosis-free patients (OR $=0.901,95 \% \mathrm{CI} 0.825$ $0.985, P=0.022)$. We additionally determined that lymphocyte count $(\mathrm{OR}=0.495,95 \%$ Cl0.260-0.943, $\mathrm{P}=0.033)$, was independent risk factors for ISR.

Conclusion: Patients with a lower PNI level at admission was at a higher risk of suffering from ISR.

\section{Keywords}

Cerebrovascular-disease, In-stent-restenosis, Prognostic-nutritional-index, Albu-min, Lymphocyte, Inflammatory response

\section{Introduction}

In patients with cerebrovascular diseases, both medications and interventional neurosurgery are the primary treatment approaches. Stent implantation, aneurysm embolization, and balloon angioplasty are all forms of percutaneous intracranial artery intervention that are implemented in certain patients. Intracranial stent implantation can lower restenosis rates via eliminating elastic retraction and negative reconstruction following balloon angioplasty. However, this approach is still limited by the incidence of in-stent restenosis (ISR), which can occur despite improvements in both implantation techniques and antiplatelet therapy in recent years.

The prognostic nutritional index (PNI) was proposed by Onodera, et al. and is computed based upon serum albumin levels and lymphocyte counts in a given patient [1]. PNI values have been successfully used as a means of gauging surgical risk and prognosis following myocardial or cerebral infarction.

At physiological concentrations, albumin is able to mediate the selective inhibition of tumor necrosis factor-induced vascular cell adhesion molecules expression. If can further suppress monocyte adhesion and transcription factor activation within endothelial cells in humans, indicating that albumin may play a key anti-inflammatory role $[2,3]$. Albumin additionally functions as a key antioxidant protein that can sequester roughly

Citation: Fang Q, Yang Y, Wei J, Fan L, Xie Z, et al. (2021) The Relationship between Reoperative Prognostic Nutritional Index Levels and Risk of In-Stent Restenosis following Cerebrovascular Bare-Metal Stent Implantation. A Case Report. Neurosurg Cases Rev 4:067. doi.org/10.23937/26434474/1710067

Accepted: May 25, 2021; Published: May 27, 2021

Copyright: (C) 2021 Fang Q, et al. This is an open-access article distributed under the terms of the Creative Commons Attribution License, which permits unrestricted use, distribution, and reproduction in any medium, provided the original author and source are credited. 
$70 \%$ of free radicals in serum. When these free radicals are not effectively neutralized, they can damage endothelial cells, stimulate vascular smooth muscle cell proliferation and intimal hyperplasia, and drive ISR and arterial lesion development [4]. There is prior evidence that reduced albumin levels upon hospital admission can independently predict stent restenosis in patients that undergo bare-metal stent implantation within the coronary artery [5]. There is also evidence that preoperative neutrophil-to-lymphocyte ratio (NLR) can predict ISR incidence in patients undergoing bare-metal stenting of the coronary artery, with lymphocyte count being negatively correlated with the incidence of cardiovascular events [6]. However, few studies have specifically evaluated the relationship between lymphocyte counts and ISR.

\section{Materials and Methods}

\section{Study population}

In total, 201 patients that had undergone successful intracranial artery stent implantation between May 2017 and May 2020 at our hospital were included in this study. Patients were excluded if they had a history of cancer, hematologic disease, severe kidney or liver disease, infectious disease, or inflammatory disease. UItimately, 141 patients that had undergone intracranial stent implantation due to acute or chronic cerebrovascular stenosis, aneurysm, or arterial dissection were included in this study cohort.

Hyperlipidemic patients were those with total cholesterol $\geq 6.0 \mathrm{mmol} / \mathrm{L}$ and/or low-density lipoprotein cholesterol $(\mathrm{LDL}-\mathrm{C}) \geq 3.49 \mathrm{mmol} / \mathrm{L}$. Patients were considered to have hypertension if their blood pressure was $\geq 140 / 90 \mathrm{mmHg}$ at the time of admission, or if they had a history of hypertension even if it was presently stable and controlled using appropriate drugs. Patients were considered to have diabetes if they had a fasting blood glucose level $\geq 7.0 \mathrm{mmol} / \mathrm{l}$, a random blood glucose level $\geq 11.1 \mathrm{mmol} / \mathrm{l}$, or a documented history of diabetes that was controlled by drugs. Our local ethics committee approved this study, and all patients provid- ed written informed consent to participate (the clinical trial number is 2021GLMU1AYJS016.

\section{Procedures}

Standard cerebral angiography and stenting were conducted via a femoral artery approach. Cerebrovascular lesions were diagnosed by experienced physicians. All patients were treated for 3-5 days prior to surgery with oral aspirin $(0.1 \mathrm{~g}$ ) and clopidogrel $(75 \mathrm{mg})$. Following femoral artery puncture, patients were intravenously administered heparin $(2 / 3 \mathrm{mg} / \mathrm{kg})$, with additional heparin being injected as needed to maintain an activated partial thromboplastin time (APTT) > 120 seconds. Successful stent implantation was defined by a lack of major complications and by a stent stenosis diameter of $<30 \%$.

\section{Laboratory assays}

Prior to stent implantation, venous blood samples were collected from each patient. The samples were analyzed for the following parameters using standard laboratory techniques: lymphocyte counts (TLC), WBC, platelet count, plasma albumin (ALB), creatinine, and fasting blood lipids (including triglycerides, total cholesterol, LDL-C, and HDL-C). PNI was calculated as follows: $\mathrm{PNI}=\mathrm{ALB}(\mathrm{g} / \mathrm{I})+5^{*} \mathrm{TLC}[7]$. ISR was diagnosed based upon a $>50 \%$ narrowing of the blood vessel at the site of stenting relative to the diameter following the most recent intervention [8].

\section{Statistical analysis}

SPSS V26.0 version was used for all data analyses. Categorical variables are given as counts with percentages and were compared via Chi-square test and Fisher's exact test, while continuous variables are means \pm standard deviations or medians with interquartile ranges and quartile ranges and were compared via Student's t-test and Mann-Whitney $U$ tests as appropriate. The goodness of fit was evaluated via the Hosmer-Lemeshow test. The relationships between variables were assessed through Pearson correlation analysis. Independent predictors of ISR were identified through multiple

Table 1: The character of clinical data of patients in the no-ISR group and ISR group.

\begin{tabular}{|l|l|l|l|}
\hline Variable & No-ISR (N = 105) & ISR (N = 36) & P \\
\hline Age, Years & $59 \pm 15$ & $63 \pm 9.0$ & 0.059 \\
\hline Hypertension & $58(55.2 \%)$ & $17(47.2 \%)$ & 0.406 \\
\hline Current smokers & $69(65.7 \%)$ & $16(52.8 \%)$ & 0.167 \\
\hline Hyperlipidemia & $57(54.3 \%)$ & $3(8.3 \%)$ & 0.308 \\
\hline Family history of stroke & $12(11.4 \%)$ & $83 \pm 17$ & 0.603 \\
\hline Heart rate, Beats/min & $81 \pm 19$ & $8(22.2 \%)$ & 0.577 \\
\hline Pre-hospital treatments & & $10(27.8 \%)$ \\
\hline DAPT & $23(21.9 \%)$ & $7(19.4 \%)$ \\
\hline Reduce blood press & $28(26.7 \%)$ & 0.968 \\
\hline Hypolipemic & $17(16.2 \%)$ & 0.897 \\
\hline
\end{tabular}

"P<0.05, with statistical difference; No ISR: No in stent restenosis; ISR: In stent restenosis; DAPT; Dual antiplatelet therapy. 
Table 2: Comparison of blood indexes between ISR group and no-ISR group.

\begin{tabular}{|l|l|l|l|}
\hline 变量 & No-ISR $(\mathrm{N}=105)$ & ISR $(\mathrm{N}=36)$ & $P$ \\
\hline WBC, $10 \% / \mathrm{L}$ & $7.3 \pm 2.2$ & $7.4 \pm 2.3$ & 0.816 \\
\hline PLT, $10^{\circ} / \mathrm{L}$ & $218 \pm 41$ & $219 \pm 46$ & 0.903 \\
\hline TLC, $10^{9} / \mathrm{L}$ & $2.1 \pm 0.1$ & $1.8 \pm 0.1$ & $<0.001^{*}$ \\
\hline ALB, $10^{\circ} / \mathrm{L}$ & $41.2 \pm 0.3$ & $40.5 \pm 0.6$ & $<0.001^{*}$ \\
\hline creatinine, umol/L & $81 \pm 21$ & $79 \pm 28$ & 0.696 \\
\hline TC, mmol/L & $3.6 \pm 1.3$ & $3.8 \pm 1.1$ & 0.410 \\
\hline LDL-C, mmol/L & $2.7 \pm 0.9$ & $2.9 \pm 0.8$ & 0.239 \\
\hline TG, mmol/L & $1.9 \pm 1.1$ & $1.8 \pm 0.9$ & 0.624 \\
\hline PNI & $51.7 \pm 0.5$ & $49.5 \pm 0.8$ & $<0.001^{*}$ \\
\hline
\end{tabular}

${ }^{*} \mathrm{P}<0.05$, with statistical difference; No ISR: No in stent restenosis; ISR: In stent restenosis; WBC: White blood cell; PLT: Platelet count; TLC: Lymphocyte count; ALB: Albumin; TC: Total cholesterol; LDL-C: Low density lipoprotein cholesterol; TG: Triglyceride; PNI: Prognostic nutritional index

Table 3: Results of binary logistics regression for ISR.

\begin{tabular}{|l|l|l|l|l|l|}
\hline Variable & $\beta$ & $\mathrm{SE}(\beta \mathrm{i})$ & Wald $X^{2}$ & OR $(95 \% \mathrm{Cl})^{\mathrm{a}}$ & $P$ 值 $(P$-value $)$ \\
\hline TLC, $10 \% / \mathrm{L}$ & -0.704 & 0.329 & 4.565 & $0.495(0.260-0.943)$ & $0.033^{*}$ \\
\hline ALB, $10 \% / \mathrm{L}$ & -0.065 & 0.060 & 1.167 & $0.937(0.832-1.055)$ & 0.280 \\
\hline PNI & -0.104 & 0.045 & 5.247 & $0.901(0.825-0.985)$ & $0.022^{*}$ \\
\hline
\end{tabular}

${ }^{*} \mathrm{P}<0.05$, with statistical difference; TLC: Lymphocyte count; ALB: Albumin; PNI: Prognostic nutritional index
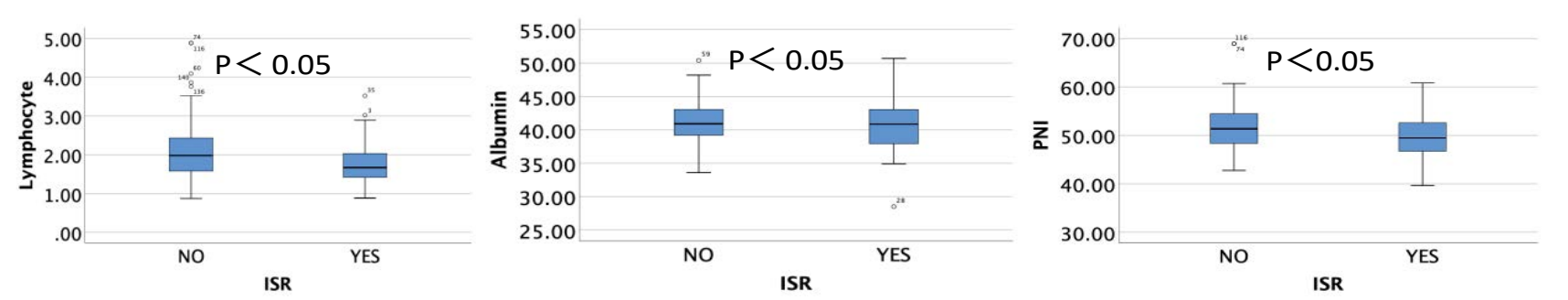

Figure 1: Comparison of TLC, ALB and PNI levels between patients with and without in-stent restenosis.

regression and logistic regression analyses. The sensitivity and specificity of PNI levels as a predictor of ISR were assessed using receiver operating characteristic (ROC) curves, which were additionally used to identify optimal PNI cut-off values for ISR prediction. $P<0.05$ was the significance threshold.

\section{Results}

There were no significant differences in the clinical baseline characteristics between the two groups (Table 1).

The results of preoperative blood tests for these patients are compiled in Table 2. Both lymphocyte counts $(P<0.001)$ and serum albumin levels $(P<0.001)$ were lower in patients in the ISR group than in the restenosis-free group. PNI levels were significantly lower in the ISR group than in the restenosis-free group (Figure 1; $49.5 \pm 0.8$ vs. $51.7 \pm 0.5, P<0.001)$. The WBC, platelet count, creatinine, and fasting blood lipids were no significantly in the two groups.

Multiple factor analysis revealed that PNI level (OR $=0.901,95 \% \mathrm{Cl}: 0.825-0.985, \mathrm{P}=0.022)$, lymphocyte count $(\mathrm{OR}=0.495,95 \% \mathrm{Cl}: 0.260-0.943, \mathrm{P}=0.033)$ were all associated with ISR incidence (Table 3).

Using the ROC curve (Figure 2), we found that the optimal cut-off PNI value at admission in the overall patient population for the prediction of ISR was 52.5, yielding a sensitivity of $80.6 \%$ and a specificity of $67.6 \%$ (AUC: $0.772,95 \% \mathrm{Cl}: 0.679-0.865, \mathrm{P}<0.05$ ).

\section{Discussion}

Herein, we evaluated the prognostic relevance of PNI levels on admission as predictors of ISR incidence in patients that underwent successful bare-metal stent implantation. Through this approach, we determined that lower PNI upon admission was associated with a greater risk of ISR development.

Stent implantation within the intracranial artery is the most prevalent form of vascular reconstructive surgery in cerebrovascular disease patients. However, the long-term efficacy of this approach is limited by ISR incidence [9]. Stent restenosis is difficult to remove and is associated with increased costs and adverse event incidence [10]. ISR exhibits a multifactorial etiology and 




Figure 2: ROC curve to evaluate the optimal cut-off value of PNI level.

may arise through a combination of inflammatory activity, extracellular matrix remodeling, granulation, and vascular smooth muscle cell (VSMC) proliferation and migration $[3,11]$.

The relationship between PNI levels and ISR incidence may be linked to ISR-associated inflammatory activity. Indeed, systemic inflammation has previously been linked to ISR incidence [3], and elevated serum levels of inflammatory cytokines have been found to be associated with an increased risk of ISR following bare-metal stent implantation. For example, Turak, et al. found that ISR incidence rose as NLR values increased in patients implanted with bare metal stents [6]. In a similar vein, Niccoli, et al. [10] determined that inflammatory status, as measured based upon CRP levels, was predictive of ISR risk following bare-metal stent implantation [12]. Serum albumin serves as a negative acute-phase protein, in that its levels fall in the context of inflammation as a consequence of decreased synthesis [13]. Oxidative stress can also shape the strength of the relationship between serum albumin and ISR incidence. ISR is associated with both oxidative stress and increased vasoactive oxygen levels, which can drive increased VSMC proliferation and consequent intimal hyperplasia, eventually it is caused by destruction of endothelial cells after stent implantation [14]. Albumin is the most abundant antioxidant protein in serum, neutralizing over $70 \%$ of free radical activity [15]. Lower serum albumin levels may thus increase oxidative stress, in turn driving the development of ISR. However, in our study, multivariate analysis showed that albumin level was not associated with in stent restenosis, this may be related to ethnic differences, and the sample size of this study is relatively small, which will affect the bias and reliability of the results. Therefore, it needs careful explanation and needs to be proved by large-scale predic- tion team research.

Lymphocytes are key mediators of immune responses [16], and low lymphocyte counts are an independent risk factor associated with cerebrovascular and cardiovascular disease incidence [17]. Kanbay, et al. also found that lower lymphocyte counts are related to dysregulated inflammatory responses and with the incidence of cerebrovascular and cardiovascular disease [18]. Lymphocytes regulate inflammatory immune responses in part via secreting cytokines [19], and there is increasingly robust evidence suggesting that chronic low-grade inflammation is a key driver of ISR pathogenesis [20].

This study has multiple limitations. For one, this was a single-center retrospective analysis of only patients implanted with bare-metal stents that experienced restenosis, and because of the small sample size, the bias factors of case selection cannot be completely excluded. In addition, our sample size was limited. Furthermore, continuous measurements were not made and so we were not able to monitor changes of time in a robust manner. As such, future prospective randomized clinical trials will be essential to assess the clinical applicability of our findings.

\section{Conclusions}

Overall, our results suggest that lower PNI values and lower lymphocyte count upon hospital admission are associated with increased ISR risk in patients undergoing bare-metal stent implantation.

\section{Statements}

\section{Acknowledgment}

We do appreciate the efforts of the intervention team in neurology department of The Affiliated Hospital of Guilin Medical University, in assisting in the collection of clinical cases.

\section{Statement of ethics}

Subjects (or their parents or guardians) have given their written informed consent. The institutional review board approved the exemption in accordance with the Ethical Guidelines for the Affiliated Hospital of Guilin Medical College, Ethical review of biomedical research involving human beings (the clinical trial number is 2021 GLMU1AYJS016.

\section{Conflict of interest statement}

The authors have no conflicts of interest to declare.

\section{Funding sources}

This research was supported by the doctoral foundation of Guilin scientific research and technology development program (No. 2017010911).

\section{Author contributions}

Q.F. and Y.Y. contributed equally to the preparation 
of this article; Q.F.: Study design, case collection, data collection, statistical analysis, and dissertation writing; Y.Y.: Shared the scientific discussion and contributed in the writing of the article; H.L.: Research guidance, study design, and case collection; J.J.W., L.G.F, Z.X.X. and Z.M.Z.: case collection.

\section{References}

1. Onodera T, Goseki N, Kosaki G (1984) Prognostic nutritional index in gastrointestinal surgery of malnourished cancer patients. Nihon Geka Gakkai zasshi 85: 1001-1005.

2. Ishida S, Hashimoto I, Seike T, Abe Y, Nakaya Y, et al. (2014) Serum albumin levels correlate with inflammation rather than nutrition supply in burns patients: A retrospective study. J Med Invest 61: 361-368.

3. Inoue T, Croce K, Morooka T, Sakuma M, Node K, et al. (2011) Vascular inflammation and repair: Implications for re-endothelialization, restenosis, and stent thrombosis. JACC Cardiovas Interv 4: 1057-1066.

4. Turak O, Canpolat U, Ozcan F, Mendi MA, Oksuz F, et al. (2014) Usefulness of preprocedural serum uric acid level to predict restenosis of bare metal stents. Am J Cardiol 113: 197-202.

5. Celik IE (2017) Preprocedural Albumin levels and risk of in-stent restenosis after coronary stenting with bare metal stent (Author's Reply). Angiology 68: 179.

6. Turak O, Ozcan F, Isleyen A, Tok D, Sokmen E, et al. (2012) Usefulness of the neutrophil-to-lymphocyte ratio to predict bare-metal stent restenosis. Am J Cardiol 110: 1405-1410.

7. Yang Y, Gao P, Song Y, Sun J, Chen X, et al. (2016) The prognostic nutritional index is a predictive indicator of prognosis and postoperative complications in gastric cancer: $A$ meta-analysis. Eur J Sur Oncol 42: 1176-1182.

8. Albuquerque FC, Levy EI, Turk AS, Niemann DB, AagaardKienitz B, et al. (2008) Angiographic patterns of Wingspan in-stent restenosis. Neurosurgery 63: 23-27.

9. Kang K, Gao F, Mo D, Yang M, Liu Y, et al. (2020) Outcome of endovascular recanalization for intracranial in-stent restenosis. J Neurointerv Surg 12: 1094-1098.

10. Niccoli G, Montone RA, Ferrante G, Crea F (2010) The evolving role of inflammatory biomarkers in risk assessment after stent implantation. J Am Coll Cardiol 56: 1783-1793.
11. Inoue T, Node K (2009) Molecular basis of restenosis and novel issues of drug-eluting stents. Circ J 73: 615-621.

12. Rahel BM, Visseren FLJ, Suttorp MJ, Plokker THW, Kelder JC, et al. (2003) Preprocedural serum levels of acute-phase reactants and prognosis after percutaneous coronary intervention. Cardiovasc Res 60: 136-140.

13. Eckart A, Struja T, Kutz A, Baumgartner A, Baumgartner T, et al. (2020) Relationship of nutritional status, inflammation, and serum albumin levels during acute illness: A prospective study. Am J Med 133: 713-722.

14. Tsuji $Y$, Koide $M$, Katsura $K$, Fujita $H$, Ishibashi-Ueda $H$, et al. (2018) In-stent restenosis with "Inflammatory" Neointima following everolimus-eluting stent implantation. Int Heart $\mathrm{J}$ 59: 1142-1145.

15. Roche M, Rondeau $P$, Singh NR, Tarnus E, Bourdon E (2008) The antioxidant properties of serum albumin. FEBS lett 582: 1783-1787.

16. Condado JF, Junpaparp $\mathrm{P}$, Binongo JN, Lasanajak $\mathrm{Y}$, Witzke-Sanz CF, et al. (2016) Neutrophil-lymphocyte ratio (NLR) and platelet-lymphocyte ratio (PLR) can risk stratify patients in transcatheter aortic-valve replacement (TAVR). Int J Cardiol 223: 444-449.

17. Bian C, Wu Y, Shi Y, Xu G, Wang J, et al. (2010) Predictive value of the relative lymphocyte count in coronary heart disease. Heart Vessels 25: 469-473.

18. Kanbay A, Kaya E, Buyukoglan H, Ozdogan N, Kaya MG, et al. (2011) Serum gamma-glutamyl transferase activity is an independent predictor for cardiovascular disease in obstructive sleep apnea syndrome. Respir Med 105: 637-642.

19. de Jager CP, van Wijk PTL, Mathoera RB, de Jongh-Leuvenink J, van der Poll T, et al. (2010) Lymphocytopenia and neutrophil-lymphocyte count ratio predict bacteremia better than conventional infection markers in an emergency care unit. Crit Care 14: R192.

20. Wang F, Li C, Ding FH, Shen Y, Gao J, et al. (2017) Increased serum TREM-1 level is associated with in-stent restenosis, and activation of TREM-1 promotes inflammation, proliferation and migration in vascular smooth muscle cells. Atherosclerosis 267: 10-18. 